\title{
Tobacco smoking in treatment-resistant schizophrenia patients is associated with impaired cognitive functioning, more severe negative symptoms, and poorer social adjustment
}

\author{
This article was published in the following Dove Press journal: \\ Neuropsychiatric Disease and Treatment \\ 6 August 2013 \\ Number of times this article has been viewed
}

\author{
Felice lasevoli \\ Raffaele Balletta \\ Valentina Gilardi \\ Sara Giordano \\ Andrea de Bartolomeis \\ Unit on Treatment Resistance \\ in Psychiatry and Laboratory of \\ Molecular and Translational Psychiatry, \\ Section of Psychiatry, Department of \\ Neuroscience, Reproductive Sciences \\ and Odontostomatology, University \\ School of Medicine 'Federico II', \\ Naples, Italy
}

\begin{abstract}
Tobacco smoking is common in schizophrenia patients. It has been reported that schizophrenia patients who are tobacco smokers have better cognitive performances compared to those who are nonsmokers. However, little is known on the effects of tobacco smoking in treatment-resistant schizophrenia (TRS) patients. The aim of this study was to compare cognitive performances, psychotic symptoms, and social adjustment in tobacco smoker TRS patients compared to nonsmoker TRS patients. Smoker and nonsmoker TRS patients did not differ in demographics and in mean daily antipsychotic dose. Smoker TRS patients had significantly higher scores than nonsmoker patients on the positive and negative syndrome scale (PANSS) and on the negative symptoms subscale. These patients also performed worse than nonsmoker patients on problem-solving cognitive domain. Social adjustment was not significantly different between the two groups. In both groups of patients, worse cognitive performances were mostly predicted by higher severity of negative symptoms. Worse performances on the verbal memory and problem-solving cognitive domains were correlated with social-functioning impairment in tobacco smoker TRS patients but not in nonsmoker ones. The results showed that tobacco smoking was not significantly associated with better cognitive performances in TRS patients, while it was significantly associated with higher negative symptoms. Even if a direct causative mechanism cannot be inferred and despite the fact that these patients may use tobacco to selfmedicate, it could be speculated that these associations may, at least partially, be related to a tobacco-smoking-induced worsening of abnormal dopamine dysfunction, which has been suggested to occur in TRS patients.
\end{abstract}

Keywords: dopamine, nicotine, psychosis, antipsychotic, cortex, refractory

\section{Introduction}

Schizophrenia patients show poorer cognitive performances and higher rates of tobacco smoking compared to non-affected individuals. ${ }^{1,2}$

Tobacco smoking in schizophrenia patients has been considered a potential attempt to self-medicate and/or to partially counteract motor and cognitive side effects of certain antipsychotic treatments. ${ }^{3,4}$ Cigarette smoking has also been described to reduce certain types of psychiatric symptoms and to lessen antipsychotic-induced side effects. ${ }^{4}$

A shared neurobiological substrate, ie, aberrant functioning of nicotinic cholinergic transmission, has been suggested to underlie both susceptibility to psychosis and vulnerability to tobacco smoking. ${ }^{5}$ Nicotine, indeed, has been demonstrated to increase dopamine release in mesolimbic and mesocortical systems, ${ }^{6-8}$ possibly 
underlying the cognitive-enhancing properties of tobacco smoking. Consistent with this view, deficits in $\alpha 7$ as well as in $\alpha 4 \beta 2$ nicotinic receptors have been associated with cognitive impairment in schizophrenia patients..$^{9,10}$ On the other hand, nicotine administration to schizophrenia patients has been reported to improve several cognitive tasks that are usually found to be impaired in schizophrenia patients, such as attention and working memory. ${ }^{11,12}$ In a recent work, acute nicotine has been found to normalize temporal aspects of sensory memory processing in a sample of twelve smokers with schizophrenia compared to twelve control smokers. ${ }^{13}$ Moreover, in a randomized double-blind, placebo-controlled study in healthy subjects stratified for their low or high propensity to auditory hallucinations/delusions, nicotine (given in a $4 \mathrm{mg}$ dose by nicotine gum) was found to revert ketamineinduced impairment of mismatch negativity. ${ }^{14}$ Nicotine was also found to improve sustained attention, as measured by the rapid visual information processing task. ${ }^{14}$

Nonsmoker schizophrenia patients were reported to perform worse than smoker patients in some cognitive tasks, such as sustained attention, processing speed, and working memory. ${ }^{15}$ Furthermore, tobacco smoking withdrawal worsened cognitive performances in tobacco smoker schizophrenia patients, ${ }^{16}$ an effect that was lost after re-exposure to tobacco.

However, tobacco smoker schizophrenia patients have also been described to experience more severe positive and global psychotic symptoms and to take higher antipsychotic doses than nonsmoker ones. ${ }^{17,18}$ These patients have been reported to suffer from poorer social adjustment compared to nonsmokers. ${ }^{18}$ Therefore, tobacco smoking may exacerbate psychosis and social defeat in patients, which may overcome nicotine-mediated putative beneficial effects on cognition.

To date, no studies have been conducted to evaluate the association between tobacco smoking and outcomes on cognitive functioning, psychotic symptoms, and social adjustment in treatment-resistant schizophrenia (TRS) patients.

TRS is defined by a sustained lack of response to at least two or three antipsychotic agents, ${ }^{19}$ despite appropriate dosage and duration of antipsychotic administration. TRS patients may be considered a special schizophrenia subpopulation, whose disease has been conceptualized as depending on distinctive dopamine dysfunctions compared to non-TRS patients, such as lack of dopamine synthesis elevation in response to antipsychotics. ${ }^{20}$

Given the reported impact of nicotine on dopamine signaling, it could be expected that cognition, symptoms, and social adjustment may be more affected in smoker TRS patients compared to non-smoker TRS ones.

Based on these considerations, we evaluated whether tobacco smoker TRS patients significantly differed from nonsmoker TRS ones in: (1) performance on selected cognitive tasks; (2) symptom severity, with specific attention on positive and negative psychotic symptoms; (3) social functioning.

We also evaluated whether these variables were interrelated and could predict cognitive performances and social functioning in the two groups of patients. As a secondary aim, we also evaluated whether tobacco smoker TRS patients were taking higher doses of antipsychotics or were administered more additional psychotropic agents.

\section{Patients and methods Study design and location}

The study was conducted according to a cross-sectional design during 2012 at the outpatient Unit for Treatment Resistance in Psychiatry of the University 'Federico II' of Naples, Italy.

\section{Participants: inclusion and exclusion criteria}

We enrolled all consecutive patients with a diagnosis of schizophrenia who met the criteria for treatment resistance. Diagnosis was made by two psychiatrists, using the Structured Clinical Interview for Diagnosis (SCID-I). Criteria for treatment resistance were derived from previously published algorithms, ie, those provided by the American Psychiatric Association. ${ }^{19}$ This algorithm states that a schizophrenia patient should be considered resistant to treatment if he/she had failed to respond to two or three trials with antipsychotic agents, given at therapeutic doses and for at least 6 weeks.

Exclusion criteria were: (1) mental retardation or severe cognitive impairment; (2) severe neurologic or systemic diseases; (3) ongoing substance abuse or addiction (excluding nicotine addiction due to cigarette smoking).

A total of 61 TRS patients were enrolled in the study. All patients were diagnosed with schizophrenia. The demographics of the sample are summarized in Table 1.

\section{Smoking status}

Patients enrolled were subdivided in two groups (smokers and nonsmokers) according to their smoking history. Nonsmokers were defined as those TRS patients who had smoked less than 100 cigarettes during their lifetime and smoked less than one cigarette each day at the moment of the evaluation. 
Table I Demographics of the sample. Demographics, mean antipsychotic doses, and mean cigarettes per day in TRS smoker and nonsmoker patients

\begin{tabular}{llll}
\hline & Smoker TRS & Nonsmoker TRS & \\
\hline Age & $37.61 \pm 9.89$ & $36.67 \pm 11.38$ & Student's $t$-test: $P=0.73 ; d f=59$ \\
Gender (M/F) & $28 / 5$ & $22 / 6$ & $\chi^{2}: 0.76$ \\
Age at disease onset & $18.89 \pm 3.06$ & $19.94 \pm 6.25$ & Student's $t$-test: $P=0.39 ; d f=59$ \\
Duration of pathology (years) & $18.72 \pm 8.87$ & $17.28 \pm 10.33$ & Student's $t$-test: $P=0.56 ; d f=59$ \\
Education (years) & $12.61 \pm 4.8 \mathrm{I}$ & $13.22 \pm 2.57$ & Student's $t$-test: $P=0.55 ; d f=59$ \\
Mean daily antipsychotic doses & $450.33 \pm 310.97$ & $442.05 \pm 258.82$ & Student's $t$-test: $P=0.91 ; d f=59$ \\
(in mg of chlorpromazine equivalent) & & & \\
Mean cigarettes per day & $12.5 \pm 6.6$ & $0.3 \pm 0.5$ & Student's $t$-test: $P<0.0000 I ; d f=59 ; t=9.73$ \\
FTND & $5.1 \pm 1.6$ & $0.2 \pm 0.1$ & Student's $t$-test: $P<0.0000 I ; d f=59 ; t=16.98$ \\
\hline
\end{tabular}

Abbreviations: FTND, Fagerstrom Test for Nicotine Dependence; M, male; F, female; TRS, treatment-resistant schizophrenia; df, degrees of freedom.

Smokers were defined as those TRS patients who smoked more than one cigarette each day and had smoked for more than 1 year. ${ }^{21,22}$ However, among those included within the smokers group, no patients were smoking less than 10 cigarettes per day at the moment of the evaluation.

Consistent with this definition, mean cigarettes per day were significantly higher in smoker TRS compared to nonsmoker TRS (Table 1).

Smoking behavior was also evaluated by the Italian version of the Fagerstrom Test for Nicotine Dependence (Table 1). ${ }^{23}$ Smoker patients had significantly higher scores at this test compared to nonsmoker ones.

All smoker patients smoked the last cigarette within 1 hour from assessments.

When subdivided into tobacco smokers $(\mathrm{n}=31)$ and nonsmokers $(\mathrm{n}=28)$, patients did not significantly differ for age, sex, education level, age at disease onset, and duration of the illness (Table 1). The two groups of patients also did not significantly differ in terms of mean daily antipsychotic doses (given as chlorpromazine equivalents, Table 1) and in the distribution of psychotropic agents prescribed (Table 2).

\section{Ethical issues}

All patients signed a written informed consent form. Patients were adequately informed of all aspects regarding the participation and the purpose of the study. All procedures carried out in the present study complied with the principles of the Declaration of Helsinki and were approved by the local Ethical Committee.

\section{Assessments}

Demographic and clinical data, as well as the type of pharmacological agent prescribed, were routinely recorded for all patients at the time of evaluation. Each patient was administered the following tests: (1) the Positive and Negative Syndrome Scale (PANSS), to assess the severity of psychotic symptoms; $;^{24}(2)$ the Personal and Social Performance (PSP) scale, to assess their ability in social performances; ${ }^{25}$ (3) the Brief Assessment of Cognition in Schizophrenia (BACS) battery of tests, to assess cognitive performance in cognitive domains that are impaired in schizophrenia patients. ${ }^{26}$

Cognitive domains assessed by the BACS were the following: ${ }^{26,27}$ Verbal Memory by the List Learning task; Working Memory by the Digit Sequencing task; Verbal Fluency by the Category Instances task; Processing Speed by the Symbol Coding task; Problem Solving by the Tower of London task. Motor Speed by the Token Motor task, which is also comprised in the BACS battery, was performed in our sample but not reported since both groups of patients had very low performance scores at this task.

Table 2 Patterns of psychotropic agents prescription. Summarization of psychotropic agent distribution among smoker and nonsmoker TRS patients

\begin{tabular}{lllllll}
\hline & FGA & SGA & AD & MS & BZ & AC \\
\hline Smoker TRS & $45.5 \%$ & $93.9 \%$ & $18.2 \%$ & $33.3 \%$ & $60.6 \%$ & 0 \\
Nonsmoker TRS & $21.4 \%$ & $96.4 \%$ & $32.1 \%$ & $28.5 \%$ & $57.1 \%$ & 0 \\
& $\chi^{2}: P>0.05, d f=1$ & $\chi^{2}: P>0.05, d f=1$ & $\chi^{2}: P>0.05, d f=1$ & $\chi^{2}: P>0.05, d f=1$ & $\chi^{2}: P>0.05, d f=1$ \\
\hline
\end{tabular}

Notes: Percentages are rates of utilization within each group.

Abbreviations: AC, anticholinergics; AD, antidepressants; BZ, benzodiazepines; FGA, first generation antipsychotics; MS, mood stabilizers; SGA, second generation antipsychotics; TRS, treatment-resistant schizophrenia; $d f$, degrees of freedom. 


\section{Statistical analysis}

Statistical analyses were carried out using JMP (version 9.0 for Mac; SAS Institute, Cary, NC, USA). Categorical data were analyzed by $\chi^{2}$ test. The unpaired Student's $t$-test was used to compare parametric data. Regression analysis by Pearson's coefficient was used to compare parametric variables. In all tests, significance was set at $P<0.05$.

\section{Results}

Tobacco smoker TRS patients exhibited significantly higher scores on the total PANSS compared to nonsmokers (Student's $t$-test: $P=0.04 ; d f=59 ; F=2.04$ ). When PANSS subscales were considered, tobacco smoker and nonsmoker TRS patients did not significantly differ on the positive and the general psychopathology subscales scores (Table 3). However, a significant difference was observed on the negative scale, where tobacco smoker TRS patients had higher scores than nonsmoker ones (Student's $t$-test: $P=0.05 ; d f=59 ; F=1.97$; Table 3 ).

Overall social functioning, as assessed by the PSP, was not significantly different in the two groups (Student's $t$-test: $P>0.05, d f=59 ; F=0.18$; Table 3$)$. No significant differences between tobacco smoker and nonsmoker TRS patients were recognized when considering single domains of social functioning (Table 3).

Tobacco smoker TRS patients performed significantly worse than nonsmoker ones on the problem solving cognitive task (Student's $t$-test: $P=0.02 ; d f=59 ; F=2.29$ ). Performances on the other cognitive tasks assesses herein (ie, verbal fluency, verbal memory, working memory, processing speed) were not found to be significantly more affected in one group compared to the other (Table 3).

We considered whether cognitive performances could be predicted by psychotic symptom severity. In smoker TRS patients, worse verbal memory $(P=0.019 ; d f=1,31$; $F=2.48)$, verbal fluency $(P=0.006 ; d f=1,31 ; F=2.95)$, and working memory $(P=0.026 ; d f=1,31 ; F=2.32)$ performances were significantly correlated with higher severity of negative symptoms (Table 4). In smoker TRS patients, poorer performances in verbal memory $(P=0.05 ; d f=1,31$; $F=2.05)$ and verbal fluency $(P=0.05 ; d f=1,31 ; F=1.98)$ domains significantly correlated with higher scores in the PANSS total (Table 4).

In nonsmoker TRS patients, poorer performances on the verbal memory $(P=0.002 ; d f=1,26 ; F=3.41)$, the verbal fluency $(P=0.037 ; d f=1,26 ; F=2.19)$, and the processing speed $(P=0.038 ; d f=1,26 ; F=2.19)$ cognitive domains significantly correlated with higher scores on the PANSS negative subscale (Table 4). Poorer performances on the problem solving cognitive domain in nonsmoker TRS patients significantly correlated with higher severity of both positive and negative symptoms $(P=0.04 ; d f=1,26 ; F=2.07$, and $P=0.001 ; d f=1,26 ; F=3.67$, respectively; Table 4).

We also evaluated whether cognitive performances could correlate with the level of social functioning. We found that, in tobacco smoker TRS patients, poorer functioning on the 'Personal and Social Relationship' domain was significantly correlated with worse performances on the verbal memory and problem solving cognitive domains $(P=0.04 ; d f=1,31$; $F=2.12$; Table 4).

Table 3 Psychopathology, social functioning, and cognitive performances in the sample

\begin{tabular}{|c|c|c|c|}
\hline & Smoker TRS & Nonsmoker TRS & \\
\hline PANSS total & $85.51 \pm 14.54$ & $77.78 \pm 14.86$ & $P=0.04 ; d f=59 ; F=2.04$ \\
\hline 'Positive Symptoms' subscale & $18.67 \pm 5.02$ & $16.44 \pm 7.17$ & $P=0.16 ; d f=59 ; F=1.42$ \\
\hline 'Negative Symptoms' subscale & $23.44 \pm 6.38$ & $20.50 \pm 5.02$ & $P=0.05 ; d f=59 ; F=1.97$ \\
\hline 'General Psychopathology' subscale & $43.39 \pm 5.77$ & $40.83 \pm 7.81$ & $P=0.15 ; d f=59 ; F=1.47$ \\
\hline PSP total & $41.22 \pm 15.91$ & $40.55 \pm 12.01$ & $P=0.86 ; d f=59 ; F=0.18$ \\
\hline 'Social Useful Activities' subscale & $3.22 \pm 1.11$ & $3.11 \pm 0.94$ & $P=0.67 ; d f=59 ; F=0.42$ \\
\hline 'Personal and Social Relationship' subscale & $3.11 \pm 0.91$ & $3.16 \pm 1.03$ & $P=0.82 ; d f=59 ; F=0.22$ \\
\hline 'Self-Care' subscale & $1.51 \pm 1.34$ & $1.11 \pm 1.08$ & $P=0.23 ; d f=59 ; F=1.19$ \\
\hline 'Disturbing and Aggressive Behavior' subscale & $0.88 \pm 0.83$ & $0.69 \pm 0.55$ & $P=0.09 ; d f=59 ; F=1.68$ \\
\hline Verbal memory & $30.83 \pm 9.92$ & $35.67 \pm 12.55$ & $P=0.09 ; d f=59 ; F=1.68$ \\
\hline Working memory & $15.17 \pm 4.47$ & $|7.5| \pm 5.3 \mid$ & $P=0.07 ; d f=59 ; F=1.86$ \\
\hline Verbal fluency & $28.44 \pm 10.52$ & $33.28 \pm 11.05$ & $P=0.08 ; d f=59 ; F=1.75$ \\
\hline Processing speed & $27.01 \pm 12.81$ & $30.72 \pm 15.34$ & $P=0.31 ; d f=59 ; F=1.03$ \\
\hline Problem solving & $8.05 \pm 4.95$ & $|I| I \pm 5.48$. & $P=0.02 ; d f=59 ; F=2.29$ \\
\hline
\end{tabular}

Notes: Smoker and nonsmoker TRS patients have been assessed by PANSS for psychotic symptoms, PSP for social adjustment, and by specific cognitive tasks for performances in selected cognitive domains. Here are summarized total scores on PANSS and PSP, as well as scores on PANSS and PSP subscales. Performance scores on cognitive domains are also reported. Significant differences using the Student's $t$-test have been given in bold.

Abbreviations: PANSS, Positive and Negative Syndrome Scale; PSP, Personal and Social Performance; TRS, treatment-resistant schizophrenia; df, degrees of freedom. 
Table 4 Correlation between cognitive performances and psychotic symptoms or social adjustment

\begin{tabular}{|c|c|c|}
\hline & Smoker TRS & Nonsmoker TRS \\
\hline \multicolumn{3}{|l|}{ Verbal memory } \\
\hline \multirow[t]{2}{*}{ PANSS total } & $P=0.05$ & NS \\
\hline & $d f=I, 3 I ; r=0.12 ; F=2.05$ & \\
\hline Positive symptom subscale & NS & NS \\
\hline \multirow[t]{2}{*}{ Negative symptom subscale } & $P=0.019$ & $P=0.002$ \\
\hline & $d f=I, 3 I ; r=0.16 ; F=2.48$ & $d f=I, 26 ; r=0.3 I ; F=3.4 I$ \\
\hline General psychopathology subscale & NS & NS \\
\hline PSP total & NS & NS \\
\hline 'Social Useful Activities' subscale & NS & NS \\
\hline \multirow[t]{2}{*}{ ‘Personal and Social Relationship’ subscale } & $P=0.04$ & NS \\
\hline & $d f=I, 3 I ; r=0.13 ; F=2.12$ & \\
\hline 'Self-Care' subscale & NS & NS \\
\hline 'Disturbing and Aggressive Behavior' subscale & NS & NS \\
\hline \multicolumn{3}{|l|}{ Working memory } \\
\hline PANSS total & NS & NS \\
\hline Positive symptom subscale & NS & NS \\
\hline \multirow[t]{2}{*}{ Negative symptom subscale } & $P=0.026$ & NS \\
\hline & $d f=I, 3 I ; r=0.15 ; F=2.32$ & \\
\hline General psychopathology subscale & NS & NS \\
\hline PSP total & NS & NS \\
\hline 'Social Useful Activities' subscale & NS & NS \\
\hline ‘Personal and Social Relationship’ subscale & NS & NS \\
\hline 'Self-Care' subscale & NS & NS \\
\hline 'Disturbing and Aggressive Behavior' subscale & NS & NS \\
\hline \multicolumn{3}{|l|}{ Verbal fluency } \\
\hline \multirow[t]{2}{*}{ PANSS total } & $P=0.05$ & NS \\
\hline & $d f=I, 31 ; r=0.1 I ; F=1.98$ & \\
\hline Positive symptom subscale & NS & NS \\
\hline \multirow[t]{2}{*}{ Negative symptom subscale } & $P=0.006$ & $P=0.037$ \\
\hline & $d f=I, 3 I ; r=0.19 ; F=2.95$ & $d f=1,26 ; r=0.16 ; F=2.19$ \\
\hline General psychopathology subscale & NS & NS \\
\hline PSP total & NS & NS \\
\hline 'Social Useful Activities' subscale & NS & NS \\
\hline ‘Personal and Social Relationship’ subscale & NS & NS \\
\hline 'Self-Care' subscale & NS & NS \\
\hline 'Disturbing and Aggressive Behavior' subscale & NS & NS \\
\hline \multicolumn{3}{|l|}{ Processing speed } \\
\hline PANSS total & NS & NS \\
\hline Positive symptom subscale & NS & NS \\
\hline \multirow[t]{2}{*}{ Negative symptom subscale } & NS & $P=0.038$ \\
\hline & & $d f=1,26 ; r=0.15 ; F=2.19$ \\
\hline General psychopathology subscale & NS & NS \\
\hline PSP total & NS & NS \\
\hline 'Social Useful Activities' subscale & NS & NS \\
\hline ‘Personal and Social Relationship’ subscale & NS & NS \\
\hline 'Self-Care' subscale & NS & NS \\
\hline 'Disturbing and Aggressive Behavior' subscale & NS & NS \\
\hline \multicolumn{3}{|l|}{ Problem solving } \\
\hline PANSS total & NS & NS \\
\hline \multirow[t]{2}{*}{ Positive symptom subscale } & NS & $P=0.04$ \\
\hline & & $d f=1,26 ; r=0.14 ; F=2.07$ \\
\hline \multirow[t]{2}{*}{ Negative symptom subscale } & NS & $P=0.00 I$ \\
\hline & & $d f=I, 26 ; r=0.34 ; F=3.67$ \\
\hline General psychopathology subscale & NS & NS \\
\hline PSP total & NS & NS \\
\hline 'Social Useful Activities' subscale & NS & NS \\
\hline
\end{tabular}


Table 4 (Continued)

\begin{tabular}{|c|c|c|}
\hline & Smoker TRS & Nonsmoker TRS \\
\hline \multirow[t]{2}{*}{ 'Personal and Social Relationship' subscale } & $P=0.04$ & NS \\
\hline & $d f=I, 3 I ; r=0.13 ; F=2.12$ & \\
\hline 'Self-Care' subscale & NS & NS \\
\hline 'Disturbing and Aggressive Behavior' subscale & NS & NS \\
\hline
\end{tabular}

Notes: Scores on cognitive tasks have been correlated with scores on PANSS total and subscales and with scores on PSP total and subscales. High scores on cognitive tasks indicate better performances. High scores on PANSS and PSP indicate high symptom level and poor social functioning, respectively. For clarity, only significant correlations were reported. All significant correlations were inverse, ie, higher scores on PANSS or PSP were associated with lower scores on cognitive tasks. Abbreviations: NS, not significant; PANSS, Positive and Negative Syndrome Scale; PSP, Personal and Social Performance; TRS, treatment-resistant schizophrenia; df, degrees of freedom.

\section{Discussion}

Our results may suggest that tobacco smoker TRS patients suffer from more severe cognitive impairment than nonsmoker TRS patients, namely poorer performances in the problem solving cognitive domain.

This finding in TRS patients is novel, considering that improved cognitive performances after exposure to nicotine ${ }^{5}$ or acute cognitive impairment after nicotine withdrawal ${ }^{16}$ have been described in the general population of schizophrenia patients. Therefore, tobacco smoking in TRS patients may not improve cognitive performances or may even worsen them. This finding does not appear to be explained by differences in antipsychotic (or other psychotropic) agents prescribed to the patients, since distribution of these agents did not significantly differ between smoker TRS and nonsmoker TRS patients.

It should however, be noted that most nicotine exposure studies in schizophrenia in the literature have some methodological differences to our study, eg, participants were allowed to smoke ad libitum during assessments or nicotine exposure was directly compared to placebo in quasiexperimental design.

Cognitive performances are regulated, at least partially, by dopamine neurotransmission in the dorsolateral prefrontal cortex, as well as by the interactions of this region with other brain regions, such as the parietal cortex, thalamus, and striatum. ${ }^{28}$ Notably, schizophrenia patients may suffer from hypodopaminergia in the prefrontal cortex, ${ }^{29}$ which may explain cognitive deficits. Tobacco smoking has been suggested to increase dopamine release by mesocortical projections as a consequence of cholinergic receptor stimulation by nicotine. ${ }^{5}$ This mechanism may explain the reported favorable effects on cognition of tobacco smoking.

Even if TRS could stem from a complex dysfunction in multiple neurotransmitters' signaling, aberrant dopamine transmission, which is different from that in non-TRS patients, has been hypothesized to explain the poor or lack of response to antipsychotics, at least in part.
The catechol- $O$-methyltransferase (COMT) Val ${ }^{158}$ allele was consistently over-represented in poor responder vs responder schizophrenia patients. ${ }^{30,31}$ Compared to $\mathrm{Met}^{158}$ homozygotes and Met/Val heterozygotes, $\mathrm{Val}^{158}$ homozygotes have greater COMT activity ${ }^{32}$ and lower dopamine levels in the cortex. Moreover, dopamine synthesis capacity may be affected in TRS patients compared to those schizophrenia patients who had responded to antipsychotic treatment. ${ }^{20}$ The relative amount and composition of D1-D2 receptor heteromers may also be different in treatment non-responders compared to responder schizophrenia patients. ${ }^{33}$ Therefore, it is possible that nicotine action on dopamine neurotransmission in TRS patients may result in neurobiological effects that are different from those described in non-TRS patients. This hypothesis may explain why tobacco smoking in TRS patients is associated with poorer cognitive performances. However, the possibility that tobacco smoking may worsen cognitive performances in TRS patients could be better explored by an experimental design that compares nicotine exposure against placebo.

It has also been observed that drugs acting as $N$-methylD-aspartate (NMDA) receptor antagonists may cause cognitive impairment and may affect sensory processing in healthy subjects. These observations support the view that NMDA receptor hypofunction may contribute to cognitive impairment, possibly also in schizophrenia patients. ${ }^{34}$ Recently, it has been demonstrated in healthy volunteers that nicotine prevents ketamine-induced impairment of mismatch negativity, an index of auditory sensory memory. ${ }^{14}$ Therefore, nicotine may help to restore cognitive deficits caused by aberrant NMDA receptor functioning, possibly explaining its beneficial use by schizophrenia patients. This study also provides indirect support to the hypothesis that nicotine receptor and NMDA receptor interplay may be crucially implicated in cognitive deficits. ${ }^{14}$ However, according to our results, nicotine may not have the same effect in TRS patients. Glutamatergic dysfunctions have been suggested to play a role in cognitive impairments and negative symptoms, ${ }^{35}$ 
which are also the core manifestations in treatment-resistant patients, and agents targeting the glutamatergic system have also been recently proposed in TRS. ${ }^{36}$ The observed association between cigarette smoking and impaired cognitive and negative symptoms in TRS patients may also depend on TRS-unique aberrant interplay between the cholinergic and glutamatergic systems.

Tobacco smoker TRS patients had significantly higher PANSS total scores compared to nonsmoker ones. This result is coherent with previous reports on the general population of schizophrenia patients. ${ }^{17}$ More pronounced overall psychotic symptoms, as measured by total PANSS score, might depend on augmented dopamine release in striatum by nicotine. ${ }^{5}$ Tobacco smoker TRS patients showed significantly higher scores on the PANSS negative subscale compared to nonsmoker ones. However, in the general population of schizophrenia patients, cigarette smoking was associated with more severe positive symptoms. ${ }^{17,18}$ Previously, severity of negative symptoms has been inversely correlated with the number of D1 receptors in the prefrontal cortex. ${ }^{37}$ Low dopamine neurotransmission in the prefrontal cortex may account for both negative and cognitive symptoms. ${ }^{38}$ It is possible that, in TRS patients, tobacco smoking results in even worse cortical hypodopaminergia by either presynaptic (eg, reduced dopamine synthesis) or postsynaptic (eg, increased COMT activity) mechanisms.

Moreover, tobacco smoker TRS patients were not taking higher antipsychotic doses and did not exhibit poorer social adjustment compared to nonsmoker ones. However, in tobacco smoker TRS patients, worse performance on the verbal memory and problem solving cognitive domains were predictive of poorer social functioning.

Our results suggest that tobacco smoking in TRS patients may be associated with a propensity to poor social functioning as a potential consequence of more severe cognitive dysfunctions and negative symptoms. These observations are consistent with a previous report in the general schizophrenia population suggesting that cigarette smoking may predispose to poorer social functioning. ${ }^{18}$

Beyond all the causative hypotheses suggested above, the possibility cannot be ruled out that tobacco smoking may represent an attempt to self-medicate in a population of TRS patients with prominent negative symptoms, cognitive deficits, and poor social functioning.

This study has a number of limitations that should be taken into account.

The high number of separate analyses carried out in this work suggests that the application of a Bonferroni test could be appropriate, in order to increase the significance level of the test and prevent the risk of type I errors. After applying Bonferroni correction, none of the reported significant differences or significant correlations were found to survive.

However, Bonferroni correction is considered an excessively conservative test, which increases the risk of type II errors, ${ }^{39,40}$ thereby strongly predisposing to the risk of false negative. Since minimizing one type of error may increase the risk of the other type of error, we chose not to apply corrections to the results (eg, Bonferroni correction), thus balancing the risk of the two types of errors. However, the possibility of false positives should also be taken into account when considering the results of this study.

The sample size is relatively small, although the eligible population represents only a subset of the general schizophrenia population. The study was carried out according to a cross-sectional design, which does not allow the investigation of cause-and-effect relationships. A prospective longitudinal design could better help determine whether tobacco smoking is a cause or a consequence of more severe cognitive impairment and more relevant negative symptoms in smoker TRS patients compared to nonsmoker ones. We did not provide a comparison with non-TRS schizophrenia patients, either smoker or nonsmoker ones. This issue was beyond the scope of the present study, but could represent an intriguing topic for further investigation.

\section{Conclusion}

Tobacco smoking was not associated with better cognitive performances in TRS patients, and possibly worsened negative symptoms, which may predispose to poorer cognitive performances and impaired social adjustment. Our results support the view that TRS patients may represent a unique schizophrenia subpopulation, with peculiar clinical and neurobiological features.

\section{Disclosure}

The authors report no conflicts of interest in this work.

\section{References}

1. Morisano D, Bacher I, Audrain-McGovern J, George TP. Mechanisms underlying the comorbidity of tobacco use in mental health and addictive disorders. Can J Psychiatry. 2009;54(6):356-367.

2. Keefe RS, Harvey PD. Cognitive impairment in schizophrenia. Handb Exp Pharmacol. 2012(213):11-37.

3. Winterer G. Why do patients with schizophrenia smoke? Curr Opin Psychiatry. 2010;23(2):112-119.

4. Kumari V, Postma P. Nicotine use in schizophrenia: the self medication hypotheses. Neurosci Biobehav Rev. 2005;29(6):1021-1034.

5. Wing VC, Wass CE, Soh DW, George TP. A review of neurobiological vulnerability factors and treatment implications for comorbid tobacco dependence in schizophrenia. Ann N Y Acad Sci. 2012;1248:89-106. 
6. Sesack SR, Grace AA. Cortico-Basal Ganglia reward network: microcircuitry. Neuropsychopharmacology. 2010;35(1):27-47.

7. Livingstone PD, Srinivasan J, Kew JN, et al. alpha7 and non-alpha7 nicotinic acetylcholine receptors modulate dopamine release in vitro and in vivo in the rat prefrontal cortex. Eur J Neurosci. 2009;29(3): $539-550$.

8. George TP, Verrico CD, Picciotto MR, Roth RH. Nicotinic modulation of mesoprefrontal dopamine neurons: pharmacologic and neuroanatomic characterization. J Pharmacol Exp Ther. 2000;295(1):58-66.

9. Freedman R, Adams CE, Leonard S. The alpha7-nicotinic acetylcholine receptor and the pathology of hippocampal interneurons in schizophrenia. J Chem Neuroanat. 2000;20(3-4):299-306.

10. Leonard S, Gault J, Hopkins J, et al. Association of promoter variants in the alpha7 nicotinic acetylcholine receptor subunit gene with an inhibitory deficit found in schizophrenia. Arch Gen Psychiatry. 2002;59(12): $1085-1096$.

11. Harris JG, Kongs S, Allensworth D, et al. Effects of nicotine on cognitive deficits in schizophrenia. Neuropsychopharmacology. 2004;29(7): 1378-1385.

12. Smith RC, Warner-Cohen J, Matute M, et al. Effects of nicotine nasal spray on cognitive function in schizophrenia. Neuropsychopharmacology. 2006;31(3):637-643.

13. Dulude L, Labelle A, Knott VJ. Acute nicotine alteration of sensory memory impairment in smokers with schizophrenia. J Clin Psychopharmacol. 2010;30(5):541-548.

14. Knott V, Shah D, Millar A, et al. Nicotine, Auditory Sensory Memory, and sustained attention in a human ketamine model of Schizophrenia: moderating influence of a hallucinatory trait. Front Pharmacol. 2012; 3:172.

15. Wing VC, Bacher I, Sacco KA, George TP. Neuropsychological performance in patients with schizophrenia and controls as a function of cigarette smoking status. Psychiatry Res. 2011;188(3):320-326.

16. Sacco KA, Termine A, Seyal A, et al. Effects of cigarette smoking on spatial working memory and attentional deficits in schizophrenia: involvement of nicotinic receptor mechanisms. Arch Gen Psychiatry. 2005;62(6):649-659.

17. Zhang XY, Chen DC, Xiu MH, et al. Cigarette smoking, psychopathology and cognitive function in first-episode drug-naive patients with schizophrenia: a case-control study. Psychol Med. Epub November 13, 2012.

18. Krishnadas R, Jauhar S, Telfer S, Shivashankar S, McCreadie RG. Nicotine dependence and illness severity in schizophrenia. $\mathrm{Br} J$ Psychiatry. 2012;201(4):306-312.

19. Lehman AF, Lieberman JA, Dixon LB, et al. Practice guideline for the treatment of patients with schizophrenia, second edition. Am J Psychiatry. 2004;161(Suppl 2):1-56.

20. Demjaha A, Murray RM, McGuire PK, Kapur S, Howes OD. Dopamine synthesis capacity in patients with treatment-resistant schizophrenia. Am J Psychiatry. 2012;169(11):1203-1210.

21. Zhang XY, Chen da C, Xiu MH, et al. Cigarette smoking and cognitive function in Chinese male schizophrenia: a case-control study. PLoS One. 2012;7(5):e36563.

22. Zhang XY, Liang J, Chen da C, et al. Cigarette smoking in male patients with chronic schizophrenia in a Chinese population: prevalence and relationship to clinical phenotypes. PLoS One. 2012;7(2):e30937.

23. Ferketich AK, Fossati R, Apolone G. An evaluation of the Italian version of the Fagerstrom Test for Nicotine Dependence. Psychol Rep. 2008; 102(3):687-694.

Neuropsychiatric Disease and Treatment

\section{Publish your work in this journal}

Neuropsychiatric Disease and Treatment is an international, peerreviewed journal of clinical therapeutics and pharmacology focusing on concise rapid reporting of clinical or pre-clinical studies on a range of neuropsychiatric and neurological disorders. This journal is indexed on PubMed Central, the 'PsycINFO' database and CAS.
24. Pancheri P, Brugnoli R, Carilli L, Delle Chiaie R, Marconi PL, Petrucci RM. Valutazione dimensionale della sintomatologia schizofrenica. Validazione della versione italiana della Scala per la valutazione dei Sintomi Positivi e Negativi (PANSS). Giornale Italiana Psicopatologia. 1995;1:60-75.

25. Morosini PL, Magliano L, Brambilla L, Ugolini S, Pioli R. Development, reliability and acceptability of a new version of the DSM-IV Social and Occupational Functioning Assessment Scale (SOFAS) to assess routine social functioning. Acta Psychiatr Scand. 2000;101(4): 323-329.

26. Anselmetti S, Poletti S, Ermoli E, et al. The Brief Assessment of Cognition in Schizophrenia. Normative data for the Italian population. Neurol Sci. 2008;29(2):85-92.

27. Keefe RS, Goldberg TE, Harvey PD, Gold JM, Poe MP, Coughenour L. The Brief Assessment of Cognition in Schizophrenia: reliability, sensitivity, and comparison with a standard neurocognitive battery. Schizophr Res. 2004;68(2-3):283-297.

28. Barch DM, Ceaser A. Cognition in schizophrenia: core psychological and neural mechanisms. Trends Cogn Sci. 2012;16(1):27-34.

29. Laruelle M, Kegeles LS, Abi-Dargham A. Glutamate, dopamine, and schizophrenia: from pathophysiology to treatment. Ann N Y Acad Sci. 2003;1003:138-158.

30. Molero P, Ortuno F, Zalacain M, Patino-Garcia A. Clinical involvement of catechol-O-methyltransferase polymorphisms in schizophrenia spectrum disorders: influence on the severity of psychotic symptoms and on the response to neuroleptic treatment. Pharmacogenomics $J$. 2007;7(6):418-426.

31. Gupta M, Bhatnagar P, Grover S, et al. Association studies of catecholO-methyltransferase (COMT) gene with schizophrenia and response to antipsychotic treatment. Pharmacogenomics. 2009;10(3):385-397.

32. Chen J, Lipska BK, Halim N, et al. Functional analysis of genetic variation in catechol-O-methyltransferase (COMT): effects on mRNA, protein, and enzyme activity in postmortem human brain. Am J Hum Genet. 2004;75(5):807-821.

33. Perreault ML, O'Dowd BF, George SR. Dopamine receptor homooligomers and heterooligomers in schizophrenia. CNS Neurosci Ther. 2011;17(1):52-57.

34. Kantrowitz JT, Javitt DC. Thinking glutamatergically: changing concepts of schizophrenia based upon changing neurochemical models. Clin Schizophr Relat Psychoses. 2010;4(3):189-200.

35. Goff DC, Coyle JT. The emerging role of glutamate in the pathophysiology and treatment of schizophrenia. Am J Psychiatry. 2001;158(9): 1367-1377.

36. de Bartolomeis A, Sarappa C, Magara S, Iasevoli F. Targeting glutamate system for novel antipsychotic approaches: relevance for residual psychotic symptoms and treatment resistant schizophrenia. Eur $J$ Pharmacol. 2012;682(1-3):1-11.

37. Okubo Y, Suhara T, Suzuki K, et al. Decreased prefrontal dopamine D1 receptors in schizophrenia revealed by PET. Nature. 1997;385(6617): 634-636.

38. Abi-Dargham A, Xu X, Thompson JL, et al. Increased prefrontal cortical $\mathrm{D}(1)$ receptors in drug naive patients with schizophrenia: a PET study with [(1)(1)C]NNC112. J Psychopharmacol. 2012;26(6):794-805.

39. Bland JM, Altman DG. Multiple significance tests: the Bonferroni method. BMJ. 1995;310(6973):170.

40. Perneger TV. What's wrong with Bonferroni adjustments. BMJ. 1998; 316(7139):1236-1238.

\section{Dovepress}

The manuscript management system is completely online and includes a very quick and fair peer-review system, which is all easy to use. Visit http://www.dovepress.com/testimonials.php to read real quotes from published authors. 